\title{
Monitoring Technologies in Acute Care Environments: A Comprehensive Guide to Patient Monitoring Technology
}

\author{
Jesse Ehrenfeld, Maxime Cannesson (Editors). Springer - New York, December 2013. \\ US\$79.99; Pages: 393; ISBN-13: 978-1461485568
}

\author{
Nicholas S. Goehner, MD • Philip E. F. Roman, MD
}

Received: 7 April 2014/ Accepted: 23 April 2014/Published online: 15 May 2014

(C) Canadian Anesthesiologists' Society 2014

Clinical monitoring is an essential component of care in modern medicine - from the emergency department to the operating suite to the intensive care unit (ICU). Much of the technology behind commonly utilized monitors operates behind the scene, from reporting raw data to computing complex algorithms that reveal the intimate realities of our patients' physiology. In Monitoring Technologies in Acute Care Environments: A Comprehensive Guide to Patient Monitoring Technology, editors Ehrenfeld and Cannesson pull back the curtain of mystery and reveal much about the history and function of commonly utilized monitors and laboratory equipment.

The 393-page monograph (softback text was reviewed, although electronic versions for eBook and Amazon Kindle are also available) is organized in eight sections, starting with an overview of the Fundamental Principles of Monitoring and moving through Hemodynamic (Section II), Respiratory (Section III), Neurologic (Section IV) and Metabolic Monitors (Section V). Section VI encompasses the gamut of unique monitors, such as fetal and coagulation monitors. The monograph then reviews the pertinent Information Technologies in the Acute Care Setting (Section VII) and concludes with New and Emerging Technologies (Section VIII). The 78 contributors are diverse in education and professions. They are

\footnotetext{
N. S. Goehner, MD

Division of Cardiothoracic Anesthesia, Department of Anesthesiology, University of Maryland School of Medicine, Baltimore, MD, USA

P. E. F. Roman, MD (ه)

Divisions of Cardiothoracic \& Transplant Anesthesia, Department of Anesthesiology, University of Maryland School of Medicine, Baltimore, MD, USA

e-mail:proman@anes.umm.edu
}

recognized experts in the field as well as clinical engineers and research scientists.

The monograph is comprehensive yet eminently readable. The chapters are concise, but each is thorough without obvious weaknesses. As can be expected from a monograph with Acute Care Environments in its title, the focus is not limited to those monitors found only in the operating suites. Admittedly, this may be a bit disappointing to non-intensivist anesthesiologists who seek to know more about the intraoperative use of only a particular technology (for example, Chapter 8, Pulmonary Artery Catheterization).

Section I begins with the history and current state of clinical monitoring and how the acute care environments are unique in medicine. The complex field of signal processing is effectively explained in a fashion that the non-engineer clinician may well comprehend. The graphics are clear, concise, and facilitate understanding. There is some repetition within the book, for example, Information Displays and Ergonomics (Chapter 5) and Decision Support and Closed-Loop Systems (Chapter 6) both contain material covered later in the monograph.

As cardiothoracic anesthesiologists, the Hemodynamic (Section II) and Respiratory Monitoring (Section III) sections were particularly interesting. While most anesthesiologists understand the function of the pulmonary artery catheter, the chapters on Non-invasive Cardiac Output Monitoring (Chapter 9) and Transpulmonary Thermodilution (Chapter 10) add the depth necessary for proper utilization of these advanced techniques. The description of Echocardiography in the Acute Care Setting (Chapter 11) is limited to transthoracic echocardiography for trauma and critical care. Inclusion of transesophageal echocardiography for non-cardiac surgery in the operating suite, recovery room, and ICU would have been welcomed. The description of both Non-invasive Arterial 
Pressure Monitoring (Chapter 12) and Hemodynamic Monitoring during Cardiopulmonary Bypass (Chapter 16) should be mandatory reading for residents and fellows.

The Respiratory Monitoring section was richly illustrated, although the quality of the diagrams varied greatly. During certain points in this section, the text was esoteric at times and difficult to follow. Nevertheless, it is crucial that anesthesiologists understand the material covered, including analysis of the pulse oximeter waveform, capnography, and ventilator settings, and time dedicated to precise reading is well rewarded.

Neuromonitoring (Section IV) was comprehensive and covered a wide range of topics, from transcranial Doppler to intracranial pressure and jugular venous saturation. Brain oxygenation monitoring is a topic of such importance that more detail should have been covered (Chapter 28), although the comparison between available monitors was well written. The chapter on evoked potentials would have benefited from including the use during descending aortic surgery and during deep hypothermic circulatory arrest.

The remainder of the monograph diverged from the principle mission. Metabolic Monitoring (Section V) encompassed seemingly unrelated topics, such as glucometrics, noninvasive hemoglobin monitors, gastric tonometry, and temperature monitoring. This section would have benefited from a discussion of CO-oximeter arterial and venous gas determination, including point-ofcare gas monitors commonly found in the ICU and emergency department. Other Forms of Monitoring in the Acute Care Environment (Section VI) included Pediatric Monitoring (Chapter 41), which is a broad topic without unique technology. Similarly, the chapter on Ultrasound (Chapter 43) was solely a description of the physics of sonography.

In summary, this text is a valuable resource for trainees who seek a more thorough understanding of some of the technology used in the hospital. The goal of being a comprehensive guide is not fully attained, however, but this should not preclude those interested from acquiring the monograph. More focus on the use of monitors in the operating suites would have been welcomed. This text would be most at home in the library of a training program in emergency medicine, anesthesiology, surgery, or critical care medicine.

Conflicts of interest None declared. 